\title{
EFICÁCIA DO DIFLUBENZURON 25\% NO CONTROLE DA HAEMATOBIA IRRITANS (DIPTERA: MUSCIDAE): DESAFIO IN VITRO E A CAMPO
}

\author{
A. Dell' Porto ${ }^{1}$, E.G.L. Hoppe ${ }^{2}$, A.G. Gomes ${ }^{3}$, R.S.S. Mata ${ }^{4}$, R.M. de S. Rocha ${ }^{4}$ \\ ${ }^{1}$ Universidade de Brasília, CP 4.508, CEP 70910-970 , Brasília, DF, Brasil. E-mail: arletedp@unb.br
}

\author{
RESUMO
}

\begin{abstract}
Avaliou-se, neste experimento, a eficácia in vitro e in vivo do diflubenzuron a $25 \%$ para uso em bovinos, no controle da infestação por Haematobia irritans. Para o teste in vitro, ovos de moscasdos-chifres foram mantidos em recipientes contendo fezes de animais não-tratados ou tratados com diflubenzuron a $25 \%$, e acompanhados até emergência dos adultos. No teste in vivo, foram utilizadas 40 fêmeas aneloradas, divididas em dois grupos: controle (C) e tratado (T) com intensidade parasitária equivalente. Durante o experimento, o grupo $\mathrm{C}$ recebeu apenas suplementação mineral, enquanto o grupo T recebeu suplementação mineral e diflubenzuron a $25 \%$. A contagem de moscas nos animais foi realizada na região dorsal, desde a nuca até as pontas da anca de cada animal, no início e ao final de um período de cinco meses. Na avaliação in vitro, o grupo controle apresentou média de emergência de $86 \%$ ( $\pm 8,4 \%)$, enquanto o grupo cultivado em fezes de bovinos tratados com diflubenzuron a $25 \%$ apresentou taxa de emergência média de $1 \%( \pm 0,2 \%)$, sendo a eficácia calculada de $98,83 \%$. No teste in vivo, não foi observada redução significativa na contagem de moscas no grupo C, porém, no grupo Thouve significativa redução da infestação por H. irritans ( $\mathrm{t}$ $=16,46, \mathrm{p}<0,0001)$. A eficácia do produto, em condições de campo, foi de 99,20\%. O diflubenzuron a $25 \%$ adicionado ao sal mineral mostrou-se eficaz contra $H$. irritans, sendo indicado para esse fim.
\end{abstract}

PALAVRAS-CHAVE: Larvicida, moscas-dos-chifres, bovinos, inibidor de desenvolvimento deinsetos.

\section{ABSTRACT}

EFFICACY OF 25\% DIFLUBENZURON FOR CONTROL OF HAEMATOBIA IRRITANS (DIPTERA: MUSCIDAE): IN VITRO AND FIELD CHALLENGES. In this experiment, the in vitro and in vivo efficacy of a $25 \%$ diflubenzuron commercial formulation were evaluated for control of Haematobia irritans infestation on cattle. For the in vitro test, horn-fly eggs were cultivated in feces from untreated or treated with $25 \%$ diflubenzuron for verification of adult emergence. In the in vivo test, 40 crossbred female bovines were divided into 2 experimental groups: Controls (C) and treated (T) with $25 \%$ diflubenzuron, with similar horn-fly infestations. In the in vitro test, the control group had a mean emergence of $86 \%( \pm 8.4 \%)$ while the group cultivated in feces from cattle treated with $25 \%$ diflubenzuron had a mean emergence rate of only $1 \%( \pm 0.2 \%)$, showing product efficacy of $98.83 \%$. In the in vivo test no significant reduction was observed in the fly count of the C group, whereas the T group showed a clear reduction in the infestation $(t=16.46, p<0.0001)$. The efficacy of the product under field conditions was $99.20 \%$. In conclusion, the formulation of $25 \%$ diflubenzuron demonstrated efficacy against $H$. irritans, and is therefore indicated for this purpose.

KEY WORDS: Larvicide, horn flies, cattle, insect growth regulator.

A mosca-dos-chifres, Haematobia irritans é considerada um dos principais problemas da pecuária. Esse parasita foi introduzido no Brasil no final da década de 1970, distribuindo-se por todo o território na década seguinte (OLIVEIRA et al., 2006).

Os prejuízos decorrentes de infestação por moscas-dos-chifres chegam a 150 milhões de dólares no Brasil (GRisi et al., 2002). As perdas econômicas são decorrentes de espoliação direta, por perda de sangue (HARRIs et al., 1974), redução da produção de leite (GARCía et al., 2001), de carne (BiANChin et al., 2004) e da taxa de prenhez (BIANChin; Alves, 2002), além de resultar em bezerros com menor peso ao desmame (Drummond et al., 1981). Com base nessas

${ }^{2}$ Universidade Estadual Paulista, Faculadade de Ciências Agrárias e Veterinárias, Departamento de Medicina Veterinária Preventiva e Reprodução Animal, Jaboticabal, SP, Brasil.

${ }^{3}$ Universidade Federal de Goiás, Instituto de Patologia Tropical e Saúde Pública, Goiânia, GO, Brasil.

${ }^{4}$ Champion Farmoquímico, Departamento Técnico, Anápolis, GO, Brasil. 
informações, torna-se óbvia a necessidade de controle químico desse artrópode, buscando minimizar o impacto do parasitismo sobre a produção animal. Entretanto, em decorrência do uso de fármacos antiparasitários surge outro problema: a deposição de resíduos em produtos de origem animal e seus derivados.

Uma alternativa ao emprego de antiparasitários com período de carência elevado é a adição de inibidores de crescimento de insetos (IGR) à dieta dos animais. As benzoilfenilureias, dentre as quais o diflubenzuron, são moléculas representantes dessa classe de inseticidas e apresentam como mecanismo de ação a inibição da síntese de quitina pelos artrópodes, consequentemente, impedindo o desenvolvimento adequado das formas imaturas (SILVA; MENDES, 2002). O uso de benzoilfenilureias, devido às suas particularidades de metabolismo e eliminação é, portanto, bastante promissor para o controle desses importantes parasitas de bovinos, já que as larvas estarão diretamente expostas ao fármaco.

Até o presente, o diflubenzuron é o IGR mais estudado e empregado contra uma variedade de insetos e pragas. Estudos revelaram que o diflubenzuron apresenta baixa toxicidade para vertebrados (KeGLEY et al., 2010), baixa deposição na musculatura de peixes (WINKALER, 2008) e ausência de resíduos tanto na carne quanto no leite (TFouni et al., 2007), não requerendo período de carência para o consumo desses produtos, e permitindo maior segurança no seu uso.

Baseado no exposto, o presente trabalho visa avaliar a eficácia in vitro e in vivo de uma formulação comercial de diflubenzuron a 25\% (Difly ${ }^{\circledR}$ ) para uso em bovinos. Para confirmar a eficácia deste fármaco sobre o desenvolvimento larvar de $H$. irritans, sem possível influência de alterações climáticas, foi realizado um experimento em condições controladas.

Insetos adultos foram coletados de animais não tratados com auxílio de redeentomológica eenviados ao Instituto de Patologia Tropical e Saúde Pública (IPTSP/GO) para obtenção de ovos em condições de laboratório. As gaiolas de oviposição eram dotadas de papel-filtro umedecido com água adoçada com sacarose, para servir como substrato para oviposição. A cada três horas, o papel era examinado a procura de ovos, que eram então avaliados quanto à viabilidade, de acordo com a coloração. Os ovos viáveis foram transferidos para recipientes com 500 $\mathrm{g}$ de fezes de animais não-tratados ou tratados com diflubenzuron a $25 \%$, em ingestão diária de $70 \mathrm{~g} /$ animal, constituindo dois grupos experimentais, Controle (C) e Tratamento (T). Cada grupo contava com quatro repetições de 50 ovos cada. Os recipientes com fezes e ovos foram mantidos em estufa de demanda bioquímica de oxigênio (B.O.D.) com temperatura constante, a $25^{\circ} \mathrm{C}$ e fotoperíodo de 12 horas, por um período de 20 dias, ao final do qual foi constatada cessação da emergência dos adultos.

A eficácia foi determinada pela relação entre o número de adultos obtidos de cada recipiente e o número inicial de ovos. Os grupos $\mathrm{C}$ e $\mathrm{T}$ foram comparados estatisticamente pelo teste de MannWhitney, com $\mathrm{P}$ ajustado em 0,05 , devido à impossibilidade de se testar a normalidade de distribuição, dado o número baixo de repetições $(n=4)$.

A eficácia do produto em condições de campo foi avaliada através de experimento realizado entre os dias 15 de setembro de 2010 a 15 de fevereiro de 2011, em duas fazendas situadas em Ouro Verde de Goiás, GO. As propriedades foram escolhidas por apresentarem grande semelhança nas condições de manejo, pasto e clima, sendo separadas por um riacho. Ambas as propriedades contavam com pasto de Brachiariabrizantha, com taxa de lotação de $4 \mathrm{UA} / \mathrm{ha}$.

Para a formação dos grupos experimentais, 40 fêmeas aneloradas, com idade entre 2 a 4 anos e peso médio de $350 \mathrm{~kg}$, foram passadas em mangueira para contagem do número total de $H$. irritans presentes na região dorsal, desde a nuca até as pontas da anca, de cada animal (Almeida et al., 2005), que consistia em contar as moscas presentes na região dorsal, desde a nuca até as pontas da anca, de cada animal. As avaliações foram realizadas sempre às $8 \mathrm{~h}$ da manhã. Os grupos experimentais, controle (C) e tratados com diflubenzuron a $25 \%(\mathrm{~T})$, foram formados por 20 fêmeas com intensidade parasitária média semelhante $(C=474,5 \mathrm{~T}=465,0)$, conforme preconizado pela portaria $\mathrm{n}^{\circ} 48$ do Ministério da Agricultura, Pecuária e Abastecimento (BRASIL, 2001).

Os grupos experimentais foram submetidos às mesmas condições de manejo e alimentação. Porém, o grupo $\mathrm{T}$ foi tratado com diflubenzuron a $25 \%$ na dosagem de $0,5 \mathrm{~g} / \mathrm{kg}$ de sal mineral pronto para uso, enquanto o grupo controle recebeu apenas sal mineral comum. O sal foi fornecido diariamente e ad libitum em ambos os grupos, com consumo médio de $70 \mathrm{~g} /$ dia por animal. Os animais dos grupos experimentais foram avaliados durante cinco meses, com a avaliação da infestação dos animais iniciando em setembro e concluindo em fevereiro.

Previamente à análise estatística desse grupo, a normalidade de distribuição dos dados foi confirmada pelo teste de D’Agostino \& Pearson. Como os grupos $\mathrm{C}$ e $\mathrm{T}$, apesar de sujeitos às mesmas condições climáticas, pertenciam a fazendas diferentes, optou-se pela comparação múltipla entre os grupos e infestações de cada grupo pelo teste $t$. Todas as análises foram realizadas com auxílio do software GraphPad Prism v. 5.04 (GRAPHPAD, 2007), com $P$ ajustado em 0,05.

O grupo controle apresentou média de emergência de $86 \%( \pm 8,4 \%)$, enquanto o grupo mantido 
em fezes de bovinos tratados com diflubenzuron a $25 \%$ apresentou taxa de emergência média de apenas $1 \%( \pm 0,2 \%)$. A eficácia calculada, tomando por base o grupo controle, foi de $98,83 \%$, de acordo com metodologia proposta por HOLDSWORTH et al., (2006). Pela análise de Mann-Whitney, constatou-se clara diferença estatística entre os grupos $(U=0,0$; $\mathrm{p}=0,0265)$.

No início do experimento não foi constatada diferença estatística entre os grupos experimentais, que apresentavam infestações médias equivalentes $(\mathrm{C}=474,5 \pm 123,8$ e $\mathrm{T}=465,0 \pm 125,2)$.

Ao final do período experimental, os animais foram novamente contidos em tronco para contagem das moscas. O grupo controle apresentou infestação média de 460 moscas-dos-chifres por animal, não sendo observada redução significativa $(t=0,7307$, $\mathrm{p}=0,4694)$ em relação ao início do experimento. Já com relação ao grupo tratado que apresentou média de 4 moscas-dos-chifres por animal, notou-se redução significativa da infestação $(\mathrm{t}=16,46, \mathrm{p}<0.0001)$. Foi constatada diferença estatística entre os tempos finais dos dois grupos experimentais $(t=15,75$; $\mathrm{p}<0,0001)$. A eficácia do produto, em condições de campo, foi de $99,20 \%$.

De acordo com Grosscurt (1978), o diflubenzuron atua comumente em todos os estágios larvais de insetos mas, em algumas espécies, os primeiros e os últimos estágios podem ser mais ou menos susceptíveis. O teste in vitro, no presente trabalho, demonstrou claramente a ação dessa molécula sobre o desenvolvimento de formas imaturas, evidenciando a sua eliminação ainda ativa nas fezes do animal tratado com essa formulação, além da boa eficácia no desafio a campo, no qual foi observada redução da contagem de moscas no grupo tratado superior a $99 \%$.

Silva; Mendes (2002) verificaram que, além de o $3^{\circ}$ estádio larval de $H$. irritans ser mais sensível à ação do diflubenzuron quando comparado com os estádios anteriores, progênies de adultos colhidos a campo e tratados com diferentes concentrações deste fármaco apresentaram malformação pupal. Por agir sobre a síntese de quitina, o diflubenzuron desenvolve nos insetos cutículas que não sustentam os músculos durante a muda, sendo incapazes de liberar suas exúvias, resultando em morte por inanição ou ruptura da nova, delicada e malformada cutícula (GROSSCURT et al., 1988), e acarretando na diminuição da população de adultos no meio.

Resultados da eficácia do diflubenzuron também foram comprovados no controle de insetos de importância em saúde pública, como Aedes aegypti (BorGes et al., 2004; MARTINS; SILVA, 2004), Culex quinquefasciatus e C. pipiens (SElf et al., 1978), Musca domestica (Miller et al., 1975; Ables, 1975) e Anopheles darlingi (CostA, 2007).
A utilização do diflubenzuron a 25\% adicionado ao sal mineral de bovinos apresentou eficácia de $98,83 \%$ e $99,20 \%$ nos testes in vitro e a campo, respectivamente, sendo indicado como alternativa para o controle desse ectoparasito.

\section{REFERÊNCIAS}

ABLES, J.R. Response of the house fly and its parasitoids to Dimilin (TH -6040). Journal of Economic Entomology, v.68, n.1, p.622-624, 1975 .

ALMEIDA, F.A.; ZOCOLLER-SENO, M.C.; BASSO, F.C.; VALÈRIO FILHO, W.V. Comparação de métodos de contagem da moscas-dos-chifres (Haematobia irritans) em diferentes regiões do corpo de bovinos da raça Guzerá e mestiço. In: CONGRESSO DE INICIAÇÃO CIENTÍFICA DA UNESP, 17., 2005, Ilha Solteira, SP. Resumos. Ilha Solteira, 2005. p.1-2.

BIANCHIN, I.; ALVES, R.G.O. Moscas-dos-chifres, Haematobia irritans: comportamento e danos em vacas e bezerros Nelore antes da desmama. Pesquisa Veterinária Brasileira, v.22, p.109-113, 2002.

BIANCHIN, I.; KOLLER W.W.; ALVES, R.G.O. Efeito da mosca-dos-chifres, Haematobia irritans (L.) (Diptera: Muscidae), no ganho de peso de bovinos Nelore. Ciência Rural, v.34, n.3, p.885-890, 2004.

BORGES, R.A.; CAVASIN, G.M.; SILVA, I.G.; ARRUDA, W.; OLIVEIRA, E.S.F.; SILVA, H.H.G.; MARTINS, F. Mortalidade e alterações morfológicas provocadas pela ação inibidora do diflubenzuron na ecdise de larvas de Aedes aegypti (Diptera, Culicidae). Revista de Patologia Tropical, v.33, n.1, p.91-104, 2004.

BRASIL. Ministério da Agricultura, Pecuária e Abastecimento. Regulamento técnico para licenciamento e/ou renovação de licença de produtos antiparasitários de uso veterinário. Portaria n.48 de 12 de maio de 1997. Brasília: MAPA, 2001. 970p.

COSTA, F.M. Avaliação da atividade inseticida do regulador de crescimento de insetos Diflubenzuron contra Anopheles darlingi Root, 1926 (Diptera, Culicidae), em condições de laboratório. 2007. 61f. Dissertação (Mestrado em Ciências Biológicas - Área de Concentração em Entomologia) Instituto Nacional de Pesquisas da Amazônia, Manaus, 2007.

DRUMMOND, R.O.; LAMBERT, G.; SMALLEY, A.E.; TERRILL, C.E. Estimated losses of livestock to pests. In: PIMENTEL, D. (Ed.). CRC Handbook of pest management in agriculture. Boca Raton: CRC, 1981. v.l, p.111-127.

GARCÍA, C.A.; SALAS, S.C.; ORTI, J.L.; VÁZQUEZ, Z.G. Dinámica poblacional de Haematobia irritans en um hato de bovinos de Soto la Marina, Tamaulipas, México. Veterinaria México, v.32, p.149-152, 2001. 
GRAPHPAD Prism. Analyse, graph and organize your data. Version 5.04. GraphPad Software, San Diego, CA, 2007.

GRISI, L.; MASSARD, C.L.; MOYA-BORJA, G.E.; PEREIRA, J.B. Impacto econômico das principais ectoparasitoses em bovinos no Brasil. A Hora Veterinária, v.21, n.125, p.8-10, 2002.

GROSSCURT, A.C. Diflubenzuron: some aspects of its ovicidal and larvicidal mode of action and evaluation of its practical possibilities. Pesticide Science, v.9, p.373-386, 1978.

GROSSCURT, A.C.; HAAR, M.T.; JONGSMA, B.;

STOKER, A. PH 70-23: A new acaracide and insecticide interfering with chitin deposition. Pesticide Science, v.22, p.51-59, 1988.

HARRIS, R.L.; MILLER, J.A.; FRAZAR, E.D. Hornflies and stableflies feeding activity. Annals of the Entomological Society of America, v.67, p.891-894, 1974.

HOLDSWORTH, P.A.; VERCRUYSSE, J.; REHBEIN, S.; PETER, R.J.; De BRUIN, C.; LETONJA, T.; GREEN, P. World Association for the Advancement of Veterinary Parasitology (W.A.A.V.P.) guidelines for evaluating the efficacy of ectoparasiticides against biting and nuisance flies on ruminants. Veterinary Parasitology, v.136, p.3-13, 2006.

KEGLEY, S.E.; HILL, B.R.; ORME S.; CHOI A.H. Pesticide Database. San Francisco: Pesticide Action Network, 2010. Disponível em: <http://www.pesticideinfo.org/Detail_ Chemical.jsp?Rec_Id=PC34807>. Acesso em: 30 mai.o 2011.

MARTINS, F.; SILVA, I.G. Avaliação da atividade inibidora do diflubenzuron na ecdise das larvas de Aedes aegypti (Linnaeus, 1762) (Diptera, Culicidae). Revista da Sociedade Brasileira de Medicina Tropical, v.37, n.2, p.135138, 2004.
MILLER, R.W.; CORLEY, C.; HILL, K.R. Feeding TH6040 to chickens: effects on larval house flies in manure and determination of residues in eggs. Journal of the Economic Entomology, v.68, p.181-182, 1975.

OLIVEIRA, A.A.A.; AZEVEDO, H.C.; MELO, C.B.; BARROS, A.T.M. de. Suscetibilidade da mosca-dos-chifres (Haemotobia irritans) a inseticidas nos tabuleiros costeiros de Alagoas, Bahia e Sergipe, Brasil. Revista Brasileira Parasitologia Veterinária, v.15, p.65-70, 2006.

SELF, L.S.; NELSON, M.J.; PANT, C.P.; USMAN, S. Field trials with two Insect Growth Regulators against Culex quinquefasciatus. Mosquito News, v.38, n.1, p.74-79, 1978.

SILVA, J.J.; MENDES, J. Effects of Diflubenzuron on immature stages of Haematobia irritans (L.) (Diptera: Muscidae) in Uberlândia, State of Minas Gerais, Brazil. Memórias do Instituto Oswaldo Cruz, v.97, p.679-682, jul. 2002.

TFOUNI, S.A.V.; FURLANI, R.P.Z.; ARAÚJO, J,.D. Avaliação da presença de resíduos em leite de vacas tratadas com diflubenzurom. Revista Instituto Adolfo Lutz, v.66, p.230-233, 2007.

WINKALER, E.U. Análises de resíduos, riscos alimentares, e variáveis bioquímicas de pacu Piaractus mesopotamicus exposto ao diflubenzuron e teflubenzuron. 2008. 60f. Tese (Doutorado em Aquicultura de Águas Continentais.) Centro de Aquicultura, Universidade Estadual Paulista, Jaboticabal, 2008.

Recebido em $4 / 7 / 11$

Aceito em 12/7/12 